\title{
Tropical Open Hurwitz Numbers
}

\author{
Benoît Bertrand (*) - Erwan Brugallé (**) - Grigory Mikhalkin (***)
}

ABSTRACT - We give a tropical interpretation of Hurwitz numbers extending the one discovered in [CJM]. In addition we treat a generalization of Hurwitz numbers for surfaces with boundary which we call open Hurwitz numbers.

Hurwitz numbers are defined as the (weighted) number of ramified coverings of a compact closed oriented surface $S$ of a given genus having a given set of critical values with given ramification profiles. These numbers have a long history, and have connections to many areas of mathematics, among which we can mention algebraic geometry, topology, combinatorics, and representation theory (see [LZ04] for example).

Here we define a slight generalization of these numbers that we call open Hurwitz numbers. To do so, we fix not only points on $S$ and ramification profiles, but also a collection of disjoint circles on $S$ and the behavior of the coverings above each of these circles. Note that the total space of the ramified coverings we consider now is allowed to have boundary components.

We also define tropical open Hurwitz numbers, and establish a correspondence with their complex counterpart. This can simply be seen as a

(*) Indirizzo dell'A.: I.U.T de Tarbes, Université Paul Sabatier, Institut de Mathématiques de Toulouse, 118 route de Narbonne, F-31062 Toulouse Cedex 9, France.

E-mail: benoit.bertrand@math.univ-toulouse.fr

(**) Indirizzo dell'A.: Universitè Pierre et Marie Curie, Paris 6, 4 place Jussieu, 75005 Paris, France.

E-mail: brugalle@math.jussieu.fr

$(* * *)$ Indirizzo dell'A.: Section de Mathématiques Université de Genève, Villa Battelle, 7 route de Drize, 1227 Carouge, Suisse.

E-mail: grigory.mikhalkin@unige.ch

Research is supported in part by the project TROPGEO of the European Research Council. Also B.B. is partially supported by the ANR-09-BLAN-0039-01, E.B. is partially supported by the ANR-09-BLAN-0039-01 and ANR-09-JCJC0097-01, and G.M. is partially supported by the Swiss National Science Foundation grants n. 125070 and 126817. 
translation in the tropical language of the computation of open Hurwitz numbers by cutting $S$ along a collection of circles. A decomposition of $S$ into pairs of pants reduces the problem to the enumeration of ramified coverings of the sphere $S^{2}$ with 3 critical values. In the particular case where all ramification points are simple, except maybe two of them, we recover the tropical computation of double Hurwitz numbers in [CJM].

This note is motivated by the forthcoming paper [BBM] where the computation of genus 0 characteristic numbers of $C P^{2}$ is reduced to enumeration of floor diagrams and computation of genus 0 open Hurwitz numbers.

We would like to thank Arne Buchholz and Hannah Markwig who pointed out an inaccuracy in the first version of the discussion at the end of the paper.

\section{Open Hurwitz numbers}

The data we need to define open Hurwitz numbers are

- $S$ an oriented connected closed compact surface;

- $\mathcal{L}$ a finite collection of disjoint smoothly embedded circles in $S$; we denote by $\stackrel{\circ}{S}$ the surface $S \backslash\left(\bigcup_{L \in \mathcal{L}} L\right)$;

- $\mathcal{P}$ be a finite collection of points in $\stackrel{\circ}{S}$;

- a number $\delta\left(S^{\prime}\right) \in Z_{\geq 0}$ associated to each connected component $S^{\prime}$ of $\stackrel{\circ}{S}$; to each circle $L \in \mathcal{L}$ which is in the closure of the connected components $S^{\prime}$ and $S^{\prime \prime}$ of $\stackrel{\circ}{S}$ (note that we may have $S^{\prime}=S^{\prime \prime}$ ), we associate the number $\gamma(L)=\left|\delta\left(S^{\prime}\right)-\delta\left(S^{\prime \prime}\right)\right|$;

- a partition $\mu(p)$ of $\delta\left(S^{\prime}\right)$ associated to each point $p \in \mathcal{P}$, where $S^{\prime}$ is the connected component of $S$ containing $p$;

- a partition $\mu(L)$ of $\gamma(L)$ associated to each circle $L \in \mathcal{L}$.

In this note we identify two continuous maps $f: S_{1} \rightarrow S$ and $f^{\prime}: S_{1}^{\prime} \rightarrow S$ if there exists a homeomorphism $\Phi: S_{1} \rightarrow S_{1}^{\prime}$ such that $f^{\prime} \circ \Phi=f$.

Now let us denote by $\mathcal{S}$ the set of all (equivalence class of) ramified coverings $f: S_{1} \rightarrow S$ where

- $S_{1}$ is a connected compact oriented surface with boundary;

- $f\left(\partial S_{1}\right) \subset \cup_{L \in \mathcal{L}} L$;

- $f$ is unramified over $S \backslash \mathcal{P}$;

- $f_{\mid f^{-1}\left(S^{\prime}\right)}$ has degree $\delta\left(S^{\prime}\right)$ for each connected component $S^{\prime}$ of $\stackrel{\circ}{S}$;

- for each point $p \in \mathcal{P}$, if $\mu(p)=\left(\lambda_{1}, \ldots, \lambda_{k}\right)$, then $f^{-1}(p)$ contains exactly $k$ points, denoted by $q_{1}, \ldots, q_{k}$, and $f$ has ramification index $\lambda_{i}$ at $q_{i}$; 
- for each circle $L \in \mathcal{L}$, if $\mu(L)=\left(\lambda_{1}, \ldots, \lambda_{k}\right)$, then $f^{-1}(L)$ contains exactly $k$ boundary components of $S_{1}$, denoted by $c_{1}, \ldots, c_{k}$, and $f_{\mid c_{i}}: c_{i} \rightarrow L$ is an unramified covering of degree $\lambda_{i}$.

Note that the Riemann-Hurwitz formula gives us

$$
\chi\left(S_{1}\right)=\sum_{S^{\prime}} \delta\left(S^{\prime}\right)\left(\chi\left(S^{\prime}\right)-\left|\mathcal{P} \cap S^{\prime}\right|\right)+\sum_{p \in \mathcal{P}} l(\mu(p))
$$

where $l(\mu(p))$ is the length of the partition $\mu(p)$ (i.e. its cardinality as a multiset of natural numbers).

DeFinition 1.1. The open Hurwitz number $H_{S}^{\delta}(\mathcal{L}, \mathcal{P}, \mu)$ is defined as

$$
H_{S}^{\delta}(\mathcal{L}, \mathcal{P}, \mu)=\sum_{f \in \mathcal{S}} \frac{1}{|A u t(f)|}
$$

where Aut $(f)$ is the set of automorphisms of $f$.



a)

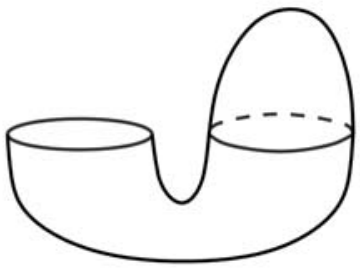

b)

Fig. 1

ExAmPLE 1.2. Let $S$ be the sphere, $L$ be a circle in $S$, and $p_{1}, p_{2}$, and $p_{3}$ three points distributed in $S$ as depicted in figure 1a. Let us also denote by $S^{\prime}$ and $S^{\prime \prime}$ the two connected components of $S \backslash L$ according to figure 1a. We define $\mu\left(p_{1}\right)=\mu\left(p_{2}\right)=\mu\left(p_{3}\right)=(2)$. The table below lists some values of $H_{S}^{\delta}(\mathcal{L}, \mathcal{P}, \mu)$ easily computable by hand. Figure 1 b depicts the only map to be taken into account in the second row of the table.

\begin{tabular}{c|c|c|c|c|c||c|}
$\delta(S)$ & $\delta\left(S^{\prime}\right)$ & $\delta\left(S^{\prime \prime}\right)$ & $\mathcal{L}$ & $\mu(\mathcal{L})$ & $\mathcal{P}$ & $H_{S}^{\delta}(\mathcal{L}, \mathcal{P}, \mu)$ \\
\hline 2 & & & $\emptyset$ & & $\left\{p_{2}, p_{3}\right\}$ & $\frac{1}{2}$ \\
\hline & 1 & 2 & $\{L\}$ & $(1)$ & $\left\{p_{2}, p_{3}\right\}$ & 1 \\
\hline & 1 & 2 & $\{L\}$ & $(1)$ & $\left\{p_{1}, p_{3}\right\}$ & 0 \\
\hline & 0 & 2 & $\{L\}$ & $(1,1)$ & $\left\{p_{2}, p_{3}\right\}$ & $\frac{1}{2}$
\end{tabular}


EXAMPLE 1.3. If $\mu(p)=(1, \ldots, 1)$, then it is clear that $H_{S}^{\delta}(\mathcal{L}, \mathcal{P}, \mu)=$ $H_{S}^{\delta}\left(\mathcal{L}, \mathcal{P}^{\prime}, \mu\right)$ where $\mathcal{P}^{\prime}=\mathcal{P} \backslash\{p\}$.

EXAMPLE 1.4. Suppose that $L \in \mathcal{L}$ bounds a disk $D$ which contains no point of $\mathcal{P}$, and that $\mu(L)=\left(\lambda_{1}, \ldots, \lambda_{k}\right)$ with $\lambda_{i} \neq 1$. Choose a point $p \in D$, define $\mathcal{L}^{\prime}=\mathcal{L} \backslash\{L\}, \mathcal{P}^{\prime}=\mathcal{P} \cup\{p\}$, and extend $\mu$ at the point $p$ by $\mu(p)=\left(1, \ldots, 1, \lambda_{1}, \ldots, \lambda_{k}\right)$ where the number of 1 we add is equal to $\delta(D)$. Then $H_{S}^{\delta}(\mathcal{L}, \mathcal{P}, \mu)=H_{S}^{\delta^{\prime}}\left(\mathcal{L}^{\prime}, \mathcal{P}^{\prime}, \mu\right)$. Here $\delta^{\prime}$ is obtained from $\delta$ by increasing it by $\gamma(L)$ over $D$.

We have to assume that $\lambda_{i} \neq 1$ for all $i$ in $\{1, \ldots, k\}$ to get this identity. Otherwise, new automorphisms of coverings might appear (e.g. the first two rows of example 1.2).

Note that the open Hurwitz number $H_{S}^{\delta}(\mathcal{L}, \mathcal{P}, \mu)$ is a topological invariant that depends only on the topological type of the triple $(S, S, \mathcal{P})$, and the functions $\delta$ and $\mu$.

In the special case where $\mathcal{L}$ is empty, we recover the usual Hurwitz numbers. In particular $\delta$ is just a positive integer number, the degree of the maps we are counting and that we denote by $d$. We simply denote Hurwitz numbers by $H_{S}^{d}(\mathcal{P}, \mu)$.

The problem of computing $H_{S}^{d}(\mathcal{P}, \mu)$ is equivalent to counting the number of some group morphisms from the fundamental group of a punctured surface to the symmetric group $\widetilde{\Xi}_{\mathcal{D}}$. Hence, Hurwitz numbers are theoretically computed by Frobenius's Formula (see for example [LZ04, Appendix, Theorems A.1.9 and A.1.10]).

EXAMPLE 1.5. If $\mathcal{P}=\left\{p_{1}, p_{2}\right\}$ is a set of two points on the sphere $S^{2}$ with $\mu\left(p_{1}\right)=\mu\left(p_{2}\right)=(d)$, then

$$
H_{S^{2}}^{d}(\mathcal{P}, \mu)=\frac{1}{d}
$$

EXAMPLE 1.6. If $\mathcal{P}=\left\{p_{1}, p_{2}, p_{3}\right\}$ is a set of three points on the sphere $S^{2}$ with $\mu\left(p_{1}\right)=\left(\lambda_{1}, \lambda_{2}\right), \mu\left(p_{2}\right)=(d)$, and $\mu\left(p_{3}\right)=(2,1, \ldots, 1)$, then

$$
H_{S^{2}}^{d}(\mathcal{P}, \mu)=\frac{1}{\left|A u t\left(\mu\left(p_{1}\right)\right)\right|}
$$

To end this section, let us mention the following nice closed formula due to Hurwitz. 
Proposition 1.7 (Hurwitz). If $\mu(p)=(2,1, \ldots, 1)$ for all $p$ in $\mathcal{P}$ except for one point $p_{0}$ for which we have $\mu\left(p_{0}\right)=\left(\lambda_{1}, \ldots, \lambda_{k}\right)$, then

$$
H_{S^{2}}^{d}(\mathcal{P}, \mu)=\frac{d^{k-3}(d+k-2) !}{\left|A u t\left(\mu\left(p_{0}\right)\right)\right|} \prod_{i=1}^{k} \frac{\lambda_{i}^{\lambda_{i}}}{\lambda_{i} !}
$$

\section{Tropical open Hurwitz numbers}

\section{1 - Tropical curves with boundary}

Given a finite graph $C$ (i.e. $C$ has a finite number of edges and vertices) we denote by $\operatorname{Vert}(C)$ the set of its vertices, by $\operatorname{Vert}^{0}(C)$ the set of its vertices which are not 1-valent, and by $\operatorname{Edge}(\mathrm{C})$ the set of its edges.

DeFinition 2.1. An irreducible tropical curve $C$ with boundary is a connected finite graph with $\mathrm{Edge}(\mathrm{C}) \neq \emptyset$ such that

- $C \backslash \operatorname{Vert}^{\infty}(C)$ is a complete metric graph for some set of 1-valent vertices $\operatorname{Vert}^{\infty}(C)$ of $C$;

- the vertices of $\operatorname{Vert}^{0}(C)$ have non-negative integer weights, i.e. $C$ is equipped with a map

$$
\begin{array}{cl}
\operatorname{Vert}^{0}(C) & \longrightarrow Z_{\geq 0} \\
v & \longmapsto g_{v}
\end{array}
$$

- any 2-valent vertex $v$ of $C$ satisfies $g_{v} \geq 1$.

If $v$ is an element of $\operatorname{Vert}^{0}(C)$, the integer $g_{v}$ is called the genus of $v$. The genus of $C$ is defined as

$$
g(C)=b_{1}(C)+\sum_{v \in \operatorname{Vert}^{0}(C)} g_{v}
$$

where $b_{1}(C)$ denotes the first Betti number of $C$. When $g(C)=b_{1}(C)$, we say that the curve $C$ is explicit.

$A$ boundary component of $C$ is a 1-valent vertex which not in $\operatorname{Vert}^{\infty}(C)$. An element of Vert $^{\infty}$ is called a leaf of $C$, and its adjacent edge is called an end of $C$.

By definition, the leaves of $C$ are at infinite distance from all the other points of $C$. A tropical curve without any boundary component is said to be closed. Our definition of tropical curves with boundary extends the defi- 
nition of tropical curves with stops introduced by Nishinou in [Nis]. We denote by $\partial C$ the set of the boundary components of $C$, and by $\operatorname{Edge}^{0}(\mathrm{C})$ the set of its edges which are not adjacent to a 1-valent vertex.

Since $O_{1}(\mathbb{R})=G L_{1}(Z)$, the data of the metric on $C$ is equivalent to the data of a $Z$-affine structure on each edge of $C$, i.e. the data of a lattice

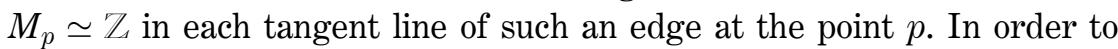
avoid unnecessary formal complications, we treat points on edges of a tropical curve as 2 -valent vertices of genus 0 in the next definition.

Definition 2.2. A continuous map $h: C_{1} \rightarrow C$ is a (non-proper) tropical morphism between the two tropical curves $C_{1}$ and $C$ if

- $h^{-1}(\partial C) \subset \partial C_{1}$;

- for any edge e of $C_{1}$, the set $h(e)$ is contained either in an edge of $C$ or in a vertex in $\operatorname{Vert}^{0}(C)$; moreover the restriction $h_{\mid e}$ is a dilatation by some integer $w_{h, e} \geq 0$ (i.e. $d h_{p}\left(M_{p}\right)=w_{h, e} M_{h(p)}$ for any $p \in e$ in the first case, and $w_{h, e}$ is obviously 0 in the second case);

- for any vertex $v$ in $\operatorname{Vert}^{0}\left(C_{1}\right)$, if we denote by $e_{1}, \ldots, e_{k}$ the edges of $C$ adjacent to $h(v)$, and by $e_{i, 1}^{\prime}, \ldots, e_{i, l_{i}}^{\prime}$ the edges of $C_{1}$ adjacent to $v$ such that $h\left(e_{i, j}^{\prime}\right) \subset e_{i}$, then one has the balancing condition

$$
\forall i, j, \quad \sum_{l=1}^{l_{i}} w_{h, e_{i, l}^{\prime}}=\sum_{l=1}^{l_{j}} w_{h, e_{j, l}^{\prime}}
$$

This number is called the local degree of $h$ at $v$, and is denoted by $d_{h, v}$;

- for any vertex $v$ in $\operatorname{Vert}^{0}\left(C_{1}\right)$, if $l$ (resp. $k$ ) denotes the number of edges e of $C$ (resp. of $C_{1}$ with $w_{f, e}>0$ ) adjacent to $h(v)$ (resp. to $v$ ) and $k>0$ then one has the Riemann-Hurwitz condition

$$
k-d_{h, v}\left(2 g_{h(v)}+l-2\right)+2 g_{v}-2 \geq 0
$$

This number is denoted by $r_{h, v}$.

The morphism $h$ is called proper if $h^{-1}(\partial C)=\partial C_{1}$.

Note that the definition implies that $h^{-1}\left(\operatorname{Vert}^{\infty}(C)\right) \subset \operatorname{Vert}^{\infty}\left(C_{1}\right)$ for a tropical morphism $h: C_{1} \rightarrow C$. The Riemann-Hurwitz condition in the previous definition comes from the classical Riemann-Hurwitz Theorem: if $S_{1}$ is a genus $g_{v}$ oriented surface with $k$ punctures, $S$ is a genus $g_{h(v)}$ oriented surface with $l$ punctures, and $f: S_{1} \rightarrow S$ is a ramified covering of degree $d_{h, v}$, then the left hand side of inequality (2) is the sum of the ramification index of all points of $S_{1}$. In particular, it is nonnegative. 
The integer $w_{h, e}$ is called the weight of the edge e with respect to $h$. When no confusion is possible, we will speak about the weight of an edge, without referring to the morphism $h$. If $w_{h, e}=0$, we say that the morphism $h$ contracts the edge $e$. The morphism $h$ is called minimal if $h^{-1}\left(\operatorname{Vert}^{\infty}(C)\right)=$ $\operatorname{Vert}^{\infty}\left(C_{1}\right)$, i.e. $h$ does not contract any end.

Two tropical morphisms $h: C_{1} \rightarrow C$ and $h^{\prime}: C_{2} \rightarrow C$ are said to be of the same combinatorial type if there exists a homeomorphism of graphs $\phi: C_{1} \rightarrow C_{2}$ (i.e. we forget about the metric on $C_{1}$ and $C_{2}$ ) such that $h=h^{\prime} \circ \phi, g(v)=g(\phi(v))$ for any vertex $v$ of $C_{1}$ and $w_{h, e}=w_{h^{\prime}, \phi(e)}$ for all $e \in \operatorname{Edge}\left(\mathrm{C}_{1}\right)$.

EXAMPLE 2.3. We depicted in figure 2a a tropical morphism from a rational tropical curve with five leaves and one boundary component to a rational curve with four ends. Three edges have weight 2 with respect to $h$. In the picture of a tropical morphism $h: C_{1} \rightarrow C$, we do not precise the lengths of edges of $C_{1}$ and $C$ since the length of edges of $C$ and the weights of edges of $C_{1}$ determine the length of edges of $C_{1}$.



a)

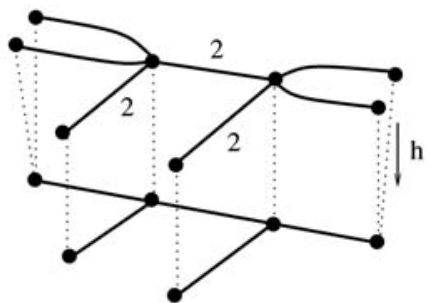

b)

Fig. 2. Representation of two tropical morphisms.

The sum of all local degrees of elements in $h^{-1}(v)$ is a locally constant function on $C \backslash h\left(\partial C_{1}\right)$; if $C^{\prime}$ is a connected component of $C \backslash h\left(\partial C_{1}\right)$ then this sum over a point of $C^{\prime}$ is called the degree of $h$ over $C^{\prime}$.

Note that for the morphism from Figure 2a we have two connected components of $C \backslash h\left(\partial C_{1}\right)$ as the boundary of $C_{1}$ consists of a single point (the only 1-valent vertex whose image is inside an edge of $C$ ). The degree over the components of $C \backslash h\left(\partial C_{1}\right)$ are 1 and 2 .

DeFinition 2.4. Let $h: C_{1} \rightarrow C$ be a tropical morphism.

$A$ subset $E$ of $C_{1}$ such that $h(E)$ is a point of $C$ is called a ramification component of $h$ if $E$ is a connected component of $h^{-1}(h(E)$ ), and contains 
either an edge in $\operatorname{Edge}^{0}\left(\mathrm{C}_{1}\right)$, or a vertex $v \in \operatorname{Vert}^{0}\left(C_{1}\right)$ with $r_{h, v}>0$, or a vertex $v \in \operatorname{Vert}^{\infty}\left(C_{1}\right)$ adjacent to an end e with $w_{h, e}>1$ (note that in such case $h(e)$ cannot be a point as otherwise $w_{h, e}=0$ ).

If $p \in C$ is such that $h^{-1}(p)$ does not contain any ramification component of $h$, we say that $h$ is unramified over $p$.

Let $v=\left(\lambda_{1}, \ldots, \lambda_{l}\right)$ be an unordered l-tuple of positive integer numbers. We say that the map $h$ has ramification profile $v$ over the leaf $v$ of $C$ if $h^{-1}(v)=\left\{v_{1}, \ldots, v_{l}\right\}$ where $v_{i}$ is a leaf of $C_{1}$ adjacent to an end of weight $\lambda_{i}$.

As in section 1, we identify two tropical morphisms $h: C_{1} \rightarrow C$ and $h^{\prime}: C_{1}^{\prime}: \rightarrow C$ if there exist two tropical isomorphisms $\Phi: C_{1} \rightarrow C_{1}^{\prime}$ and $\phi: C \rightarrow C$ such that $\phi$ restricts to the identity map on $\mathcal{Q}$ and $\mathcal{R}$, and $h^{\prime} \circ \Phi=\phi \circ h$. An automorphism $\phi$ of a tropical morphism $h: C_{1} \rightarrow C$ is a tropical isomorphism $\phi: C_{1} \rightarrow C_{1}$ such that $h \circ \phi=h$.

\section{2 - Definition of tropical open Hurwitz numbers}

Similarly to section 1 , we start with the following data

- $C$ a closed explicit tropical curve with $\operatorname{Vert}^{0}(C) \neq \emptyset$;

- $\mathcal{R}$ a finite collection of points in $C \backslash \operatorname{Vert}(C)$ such that any connected component of the set $C \backslash \mathcal{R}$, denoted $C$, contains a vertex of $C$;

- $\mathcal{Q}$ be a finite collection of points in $\operatorname{Vert}^{\infty}(C)$;

- a number $\delta\left(C^{\prime}\right) \in Z_{\geq 0}$ associated to each connected component $C^{\prime}$ of $\stackrel{\circ}{C}$; to each point $q \in \mathcal{R}$ which is in the closure of the connected components $C^{\prime}$ and $C^{\prime \prime}$ of $\stackrel{\circ}{C}$, we associate the number $\gamma(q)=\left|\delta\left(C^{\prime}\right)-\delta\left(C^{\prime \prime}\right)\right|$;

- a partition $v(q)$ of $\delta\left(C^{\prime}\right)$ associated to each point $q \in \mathcal{Q}$, where $C^{\prime}$ is the connected component of $C$ containing $q$;

- a partition $v(q)$ of $\gamma(q)$ associated to each point $q \in \mathcal{R}$.

We denote by $\mathcal{S}^{\text {T }}$ the set of all minimal tropical morphisms $h: C_{1} \rightarrow C$ such that

- $C_{1}$ is a tropical curve with boundary;

- $h\left(\partial C_{1}\right) \subset \mathcal{R}$;

- $h$ is unramified over $C \backslash \mathcal{Q}$;

- $h_{\mid h^{-1}\left(C^{\prime}\right)}$ has degree $\delta\left(C^{\prime}\right)$ for each connected component $C^{\prime}$ of $\stackrel{\circ}{C}$;

- for each point $q \in \mathcal{Q}$, the map $h$ has ramification profile $v(q)$ over $q$;

- for each point $q \in \mathcal{R}$, if $v(q)=\left(\lambda_{1}, \ldots, \lambda_{k}\right)$, the set $h^{-1}(q)$ contains exactly $k$ boundary components of $C_{1}$, denoted by $c_{1}, \ldots, c_{k}$, and $c_{i}$ is adjacent to an edge of $C_{1}$ of weight $\lambda_{i}$. 
Note that the fact that $h$ is minimal and unramified over $C \backslash \mathcal{Q}$ implies that $h\left(\operatorname{Vert}^{0}\left(C_{1}\right)\right) \subset \operatorname{Vert}^{0}(C)$ and that $h$ does not contract any edge of $C_{1}$. In particular the set $\mathcal{S}^{\mathrm{T}}$ is finite. Moreover the length of edges of $C_{1}$ are completely determined by the combinatorial type of $h$. In other words, the following lemma holds.

Lemma 2.5. Two distinct elements of $\mathcal{S}^{T}$ have distinct combinatorial types.

As usual in tropical geometry, a tropical morphism $h: C_{1} \rightarrow C$ in $\mathcal{S}^{T}$ should be counted with some multiplicity. Given $v$ a vertex in $\operatorname{Vert}^{0}\left(C_{1}\right)$ such that $h(v)$ is adjacent to the edges $e_{1}, \ldots, e_{k_{v}}$ of $C$, we choose a configuration $\mathcal{P}^{\prime}=\left\{p_{1}^{\prime}, \ldots, p_{k_{n}}^{\prime}\right\}$ of $k_{v}$ points on the sphere $S^{2}$, and we define $\mu^{\prime}\left(p_{i}^{\prime}\right)$ as the partition of $d_{h, v}$ defined by $h$ at $v$ above the edge $e_{i}$ (cf the balancing condition (1)).

Definition 2.6. The multiplicity of $h: C_{1} \rightarrow C$ is defined as

$$
m(h)=\frac{1}{|A u t(h)|} \prod_{e \in \mathrm{Edge}^{0}\left(\mathrm{C}_{1}\right)} w_{h, e} \prod_{v \in \operatorname{Vert}^{0}\left(C_{1}\right)}\left(\prod_{i=1}^{k_{v}} \mid \operatorname{Aut}\left(\mu^{\prime}\left(p_{i}^{\prime}\right) \mid\right) H_{S^{2}}^{d_{h, v}}\left(\mathcal{P}^{\prime}, \mu^{\prime}\right)\right.
$$

The tropical open Hurwitz number ' $T H_{C}^{\delta}(\mathcal{R}, \mathcal{Q}, v)$ is defined as

$$
{ }^{\top} H_{C}^{\delta}(\mathcal{R}, \mathcal{Q}, v)=\sum_{h \in \mathcal{S}^{T}} m(h)
$$

As in section 1 , if $\mathcal{R}=\emptyset$ then $\delta$ is a number denoted by $d$, and we denote by ${ }^{\top} T H_{C}^{d}(\mathcal{Q}, v)$ the corresponding tropical (closed) Hurwitz number.

EXAMPLE 2.7. Let $h: C_{1} \rightarrow C$ be the tropical morphism depicted in figure 2a. It is the tropical analog of the map considered in figure 1. Let $q_{1}$ be the image of the boundary component of $C_{1}$, and $q_{2}$ and $q_{3}$ be the leaves of $C$ which are image of a leaf of $C_{1}$ adjacent to an edge of weight 2. We denote by $C^{\prime}$ (resp. $C^{\prime \prime}$ ) the connected component of $C \backslash\left\{q_{1}\right\}$ which does not contain (resp. contains) $q_{2}$ and $q_{3}$, and we define $\delta\left(C^{\prime}\right)=1$, $\delta\left(C^{\prime \prime}\right)=2, v\left(q_{1}\right)=(1)$, and $v\left(q_{2}\right)=v\left(q_{3}\right)=(2)$. To compute $\mathbb{T} H_{C}^{\delta}(\mathcal{R}, \mathcal{Q}, v)$, the morphism of figure $2 \mathrm{a}$ is the only one to consider and it has multiplicity 1 so $T H_{C}^{\delta}(\mathcal{R}, \mathcal{Q}, v)=1$ (see the second row of the table in example 1.2). 
EXAMPLE 2.8. Let $C$ be a closed rational curve with four leaves. We set $\mathcal{Q}=\operatorname{Vert}^{\infty}(C)$ and $v(q)=(3)$ for $q \in \mathcal{Q}$. Then according to figure 3, we have ${ }^{\top} H_{C}^{3}(\mathcal{Q}, v)=1$.

Indeed, for the first morphism of Figure 3 we have the (classical) Hurwitz numbers at the inner vertices equal to $\frac{1}{3}$ each. In the same time the group of local automorphism at each vertex is the symmetric group $\widetilde{\Xi}_{3}$, so its order is 6 . Finally the group of automorphisms of the morphism itself is also $\widetilde{\Xi}_{3}$. Using Definition 2.6 we get $\frac{1}{6}\left(\frac{6}{3}\right)^{2}=\frac{2}{3}$ as the multiplicity.

For the second morphism we have local Hurwitz numbers at the inner vertices equal to $\frac{1}{3}$ again. There are no local or global automorphisms, but there is an inner edge of weight 3 . Thus we get $3\left(\frac{1}{3}\right)^{2}=\frac{1}{3}$ as the multiplicity.


Fig. 3

EXAMPLE 2.9. Let $C$ be a genus 2 explicit tropical curve with two leaves and whose combinatorial type is as in figure 4 . We set $\mathcal{Q}=\operatorname{Vert}^{\infty}(C)$ and $v(q)=(2)$ for $q \in \mathcal{Q}$. Then according to figure 4 , we have $T H_{C}^{2}(\mathcal{Q}, v)=8$.

ExAMPLE 2.10. Suppose that $q \in \mathcal{R}$ is on an end with an adjacent leaf $q^{\prime}$ not in $\mathcal{Q}$, and that $v(q)=\left(\lambda_{1}, \ldots, \lambda_{k}\right)$ with $\lambda_{i} \neq 1$. Define $\mathcal{R}^{\prime}=\mathcal{R} \backslash\{q\}$, $\mathcal{Q}^{\prime}=\mathcal{Q} \cup\left\{q^{\prime}\right\}$, and $v\left(q^{\prime}\right)=\left(1, \ldots, 1, \lambda_{1}, \ldots, \lambda_{k}\right)$ where the number of 1 we add is equal to $\delta\left(\left(q q^{\prime}\right)\right)$. Then $H_{\delta}^{\top}(C, \mathcal{R}, \mathcal{Q}, v)=H_{\delta^{\prime}}^{\top}\left(C, \mathcal{R}^{\prime}, \mathcal{Q}^{\prime}, v\right)$, where $\delta^{\prime}$ is obtained from $\delta$ by increasing it by $\gamma(q)$ over $\left(q q^{\prime}\right)$. This is the tropical counterpart of the identity described in example 1.4. Note that as in example 1.4, we have to assume that $\lambda_{i} \neq 1$ for all $i$ in $\{1, \ldots, k\}$ otherwise new automorphisms of coverings might appear (e.g. Figures $2 a$ and $b$ which are the tropical analogs of the morphisms corresponding to the first two rows of example 1.2). 



$m(h)=1$

$m(h)=1$

$m(h)=2$

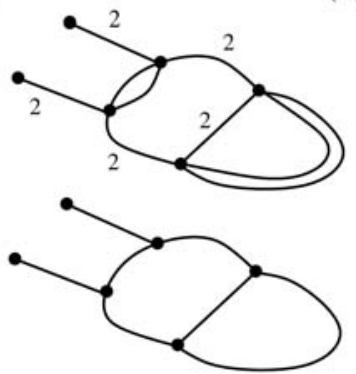

$m(h)=2$

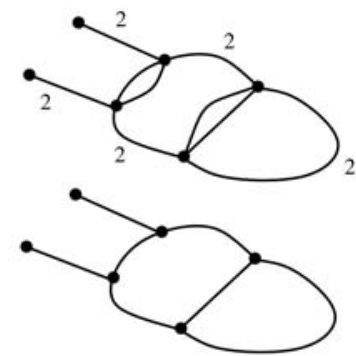

$m(h)=2$

Fig. 4
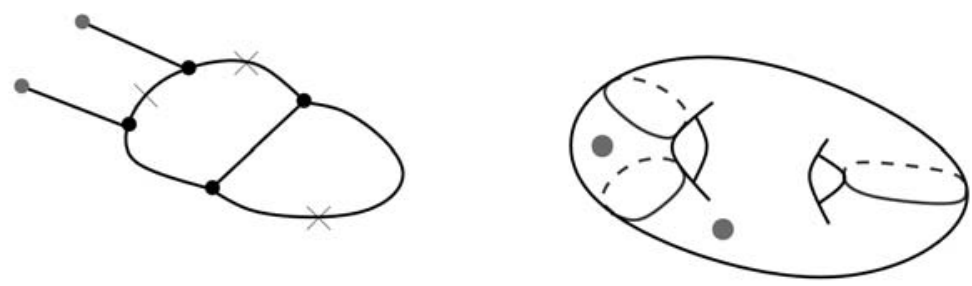

Fig. 5. A tropical curve $C$ is depicted on the left and the corresponding surface $S$ on the right. The two leaves of $C$ are elements of $\mathcal{Q}$ and correspond to elements of $\mathcal{P}$ (depicted by dots) on $S$ while crosses on $C$ represent points of $\mathcal{R}$ and correspond to the circles of $\mathcal{L}$ pictured on $S$.

Let us relate these tropical open Hurwitz numbers to the open Hurwitz numbers we defined in section 1 . Let $C$ be a tropical curve as in definition 2.6 with the data introduced at the beginning of this subsection. Let $S$ be an oriented connected compact closed surface whose genus is the genus of $C$. We choose a collection $\mathcal{L}=\left\{L_{q}\right\}_{q \in \mathcal{R}}$ of disjoint smoothly embedded 
circles in $S$ such that there is a natural correspondence $C^{\prime} \rightarrow S_{C^{\prime}}^{\prime}$ between the connected components of $\stackrel{\circ}{C}$ and $\stackrel{\circ}{S}$ which preserves incidence relations and such that $b_{1}\left(C^{\prime}\right)=g\left(C_{S^{\prime}}^{\prime}\right)$. For each point $q \in \mathcal{Q}$, we choose a point $p_{q} \in S_{C^{\prime}}^{\prime}$ where $C^{\prime}$ is the connected component of $C$ containing $q$, such that $p_{q} \neq p_{q^{\prime}}$ for $q \neq q^{\prime}$ (see figure 5 for an example). Finally we define $\mathcal{P}=\cup_{q \in \mathcal{Q}}\left\{p_{q}\right\}, \delta\left(S_{C^{\prime}}^{\prime}\right)=\delta\left(C^{\prime}\right), \mu\left(L_{q}\right)=v(q)$, and $\mu\left(p_{q}\right)=v(q)$.

Theorem 2.11. For any $\delta, \mathcal{R}, \mathcal{Q}$, and $v$, one has

$$
{ }^{\top} H_{C}^{\delta}(\mathcal{R}, \mathcal{Q}, v)=H_{S}^{\delta}(\mathcal{L}, \mathcal{P}, \mu)
$$

PRoof. The proof is quite straightforward: there exists a natural surjection $\phi: \mathcal{S} \rightarrow \mathcal{S}^{\mathrm{T}}$, and for any element $h: C_{1} \rightarrow C$ we have

$$
m(h)=\sum_{f \in \phi^{-1}(h)} \frac{1}{|A u t(f)|}
$$

Note that according to examples 1.4 and 2.10, we may suppose that $\operatorname{Vert}^{\infty}(C)=\mathcal{Q}=\mathcal{P}=\emptyset$.

Let us construct the map $\phi$. For this we choose a configuration $\mathcal{R}^{\prime}$ of points lying in the edges of $C$ such that each edge in $\operatorname{Edge}^{0}(C)$ contains exactly one point of $\mathcal{R} \cup \mathcal{R}^{\prime}$, and we define $\stackrel{\circ}{0}_{0}=C \backslash\left(\mathcal{R} \cup \mathcal{R}^{\prime}\right)$. For each point $q$ in $\mathcal{R}^{\prime}$, we choose a smoothly embedded circle $L_{q}$ in $S$ such that $L_{q} \cap L_{q^{\prime}}=\emptyset$ if $q \neq q^{\prime} \in \mathcal{R} \cup \mathcal{R}^{\prime}$, such that there is a natural correspondence $C^{\prime} \rightarrow S_{C^{\prime}}^{\prime}$ between the connected components of $\stackrel{\circ}{C}_{0}$ and $\stackrel{\circ}{S}_{0}=S \backslash\left(\mathcal{L} \cup_{q \in \mathcal{R}^{\prime}} L_{q}\right)$ which preserves incidence relations, and such that all connected components of $S_{0}$ have genus 0 . Note that $C$ is a realisation of the dual graph of the decomposition of $S$ induced by $\left(\mathcal{L} \cup_{q \in \mathcal{R}^{\prime}} L_{q}\right)$, and that a connected component of $S_{0}$ cannot be a disk since $\operatorname{Vert}^{\infty}(C)=\emptyset$.

Let $f: S_{1} \rightarrow S$ be an element of $\mathcal{S}$, and let us denote $\stackrel{\circ}{S}_{1}=f^{-1}\left(\stackrel{\circ}{S}_{0}\right)$. We construct a graph $C_{1}$ in the following way

- to each connected component $S_{1}^{\prime}$ of $\stackrel{\circ}{S}_{1}$ corresponds a vertex $v_{S_{1}^{\prime}}$ in $\operatorname{Vert}^{0}\left(C_{1}\right)$; we set $g_{v_{S_{1}^{\prime}}}$ to be equal to the genus of $S_{1}^{\prime}$;

- to each circle $L$ in $f_{\circ}^{-1}\left(\bigcup_{q \in \mathcal{R} \cup \mathcal{R}^{\prime}} L_{q}\right) \backslash \partial S_{1}$ adjacent to the connected components $S_{1}^{\prime}$ and $S_{1}^{\prime \prime}$ of $\stackrel{\circ}{S}_{1}$ corresponds an edge $e_{L}$ in $\operatorname{Edge}{ }^{0}\left(\mathrm{C}_{1}\right)$ joining $v_{S_{1}^{\prime}}$ and $v_{S_{1}^{\prime \prime}}$;

- to each connected component $L$ of $\partial S_{1}$ adjacent to the connected component $S_{1}^{\prime}$ of $\stackrel{\circ}{S}_{1}$ correspond a boundary point $v_{L}$ in $\partial C_{1}$ and an edge $e_{L}$ joining $v_{L}$ to $v_{S_{1}^{\prime \prime}}$. 
For each circle $L$ in $f^{-1}\left(\bigcup_{q \in \mathcal{R} \cup \mathcal{R}^{\prime}} L_{q}\right)$, we denote by $w\left(e_{L}\right)$ the degree of the unramified covering $f_{\mid L}: L \rightarrow f(L)$. There exists a unique tropical structure on $C_{1}$ and a unique tropical morphism $h: C_{1} \rightarrow C$ such that $h\left(v_{S_{1}^{\prime}}\right)=v \Leftrightarrow f\left(S_{1}^{\prime}\right)=S_{v}^{\prime}, \quad h\left(e_{L}\right) \subset e \Leftrightarrow f(L)=L_{q}$ where $q=\left(\mathcal{R} \cup \mathcal{R}^{\prime}\right) \cap e$, and

$$
w_{h, e_{L}}=w\left(e_{L}\right) \quad \forall e_{L} \in \operatorname{Edge}\left(\mathrm{C}_{1}\right)
$$

We define $\phi(f)=h$.

Now it remains us to prove that $m(h)=\sum_{f \in \phi^{-1}(h)} \frac{1}{|A u t(f)|}$ for any $h: C_{1} \rightarrow C$ in $\mathcal{S}^{T}$. For this, we reconstruct elements in $\phi^{-1}(h)$ step by step, taking care of automorphisms.

The tropical curve $C_{1}$ induces the structure of a reducible tropical curve on the topological closure $C_{1}$ of $C_{1} \backslash h^{-1}\left(\mathcal{R} \cup \mathcal{R}^{\prime}\right)$. Note that to any vertex $v$ in $\operatorname{Vert}^{0}\left(C_{1}\right)$ corresponds a connected component of $\stackrel{\circ}{C}_{1}$. The tropical morphism $h: C_{1} \rightarrow C$ induces a reducible tropical morphism $\stackrel{\circ}{h}: \stackrel{\circ}{C}_{1} \rightarrow C$. For each vertex $v$ in $\operatorname{Vert}^{0}\left(C_{1}\right)$ adjacent to the edges $e_{1}, \ldots, e_{k_{v}}$, we choose an unramified covering $f_{v}: S_{1, v}^{\prime} \rightarrow S_{f(v)}^{\prime}$ of degree $d_{h, v}$ where $S_{1, v}^{\prime}$ is a surface with boundary components $L_{1}, \ldots, L_{k_{v}}$ of genus $g_{v}$, and $f_{v \mid L_{i}}$ is a degree $w_{f, e_{i}}$ unramified covering from $L_{i}$ to $L_{q}$ where $q=\left(\mathcal{R} \cup \mathcal{R}^{\prime}\right) \cap e_{i}$. Let us set $\stackrel{\circ}{S}_{1}=\cup_{v \in \operatorname{Vert}^{0}\left(C_{1}\right)} S_{1, v}^{\prime}$, and let $\stackrel{\circ}{f}: \stackrel{\circ}{S}_{1} \rightarrow S$ be the map defined by ${\stackrel{\circ}{\mid S_{1, v}^{\prime}}}_{f}=f_{v}$ for any $v$ in $\operatorname{Vert}^{0}\left(C_{1}\right)$. The union of all maps $\stackrel{\circ}{f}$ constructed in this way (up to equivalence) is a finite set $A_{0}$, and we clearly have (in the notation of Definition 2.6)

$$
\sum_{f \in A_{0}} \frac{1}{|A u t(\stackrel{\circ}{f})|}=\frac{1}{|A u t(\stackrel{\circ}{h})|} \prod_{v \in \operatorname{Vert}^{0}\left(C_{1}\right)}\left(\prod_{i=1}^{k_{v}}\left|A u t\left(\mu^{\prime}\left(p_{i}^{\prime}\right)\right)\right|\right) H_{S^{2}}^{d_{h, v}}\left(\mathcal{P}^{\prime}, \mu^{\prime}\right) .
$$

To get an element $f: S_{1} \rightarrow S$ of $\mathcal{S}$, it remains to glue all the coverings $f_{v}$ according to the edges in Edge ${ }^{0}\left(\mathrm{C}_{1}\right)$. Suppose we already have performed these gluings according to $s$ edges in $\operatorname{Edge}^{0}\left(\mathrm{C}_{1}\right)$ and obtained a set $A_{s}$ of coverings of $S$, and that we want now to glue elements of $A_{s}$ along the edge $e$ in Edge ${ }^{0}\left(\mathrm{C}_{1}\right)$. Since we can identify in exactly $d$ different ways two degree $d$ unramified coverings of the circle by the circle, we have $w_{h, e}$ different ways to perform this gluing given an element $\widetilde{f}: \widetilde{S}_{1} \rightarrow S$ of $A_{s}$. We denote by $\widetilde{f}_{1}, \ldots, \widetilde{f}_{w_{h, e}}$ the coverings of $S$ constructed in this way. Let $i=1, \ldots, w_{h, e}$. Any automorphism $\psi \in \operatorname{Aut}(\widetilde{f})$ fixing globally the two boundary components of $\widetilde{S}_{1}$ corre- 
sponding to $e$ extends to an equivalence of coverings $\psi_{i}: \widetilde{f}_{i} \rightarrow \widetilde{f}_{j}$ for some $j$. The homeomorphism $\psi_{i}$ is in $\operatorname{Aut}\left(\widetilde{f}_{i}\right)$ if $i=j$, and identifies the two coverings $\widetilde{f}_{i}$ et $\widetilde{f}_{j}$ otherwise. Hence at the end of this gluing procedure, we obtain a set $A_{\left|\operatorname{Edge}^{0}\left(\mathrm{C}_{1}\right)\right|}$ and we have

$$
\sum_{f \in A_{\left|\mathrm{Edge}^{0}\left(\mathrm{C}_{1}\right)\right|}} \frac{1}{|A u t(f)|}=m(h) .
$$

According to Lemma 2.5, the set $A_{\left|\operatorname{Edge}^{0}\left(\mathrm{C}_{1}\right)\right|}$ is exactly $\phi^{-1}(h)$ so the Theorem is proved.

We can allow points with ramification profile $v(q)=(2,1, \ldots, 1)$ in $C \backslash \operatorname{Vert}^{\infty}(C)$, and recover in this way results from [CJM]. Suppose that $C$ is trivalent, and that there exists $q \in \mathcal{Q}$ with $v(q)=(2,1, \ldots, 1)$. Let $e$ be the end of $C$ adjacent to $q$, and $v$ be the other vertex adjacent to $e$. We denote by $e_{1}, \ldots, e_{k}$ the edges of $C_{1}$ in $h^{-1}(e)$, and by $\left\{v_{1}, \ldots, v_{l}\right\}=h^{-1}(v)$. By assumption on $v(q)$, one of the edges $e_{j}$, say $e_{1}$, has weight 2 while the other edges $e_{j}$ have weight 1 . Suppose that $e_{1}$ is adjacent to $v_{1}$. The Riemann-Hurwitz condition implies that all vertices $v_{j}$ are $\left(d_{h, v_{j}}+2\right)$ valent vertices of $C_{1}$, so the vertex $v_{1}$ (resp. $v_{j}, j \geq 2$ ) has exactly 3 (resp. 2) adjacent edges not mapped to $e$. Now according to Examples $1.3,1.5$, and 1.6 we have

$$
\prod_{j=1}^{l}\left(\prod_{i=1}^{3} \mid A u t\left(\mu^{\prime}\left(p_{i}^{\prime}\right) \mid\right) \mathbb{T}_{C}^{d_{h, v_{j}}}\left(\mathcal{P}^{\prime}, \mu^{\prime}\right)=\left(d_{h, v_{1}}-2\right) ! \prod_{j=2}^{l} \frac{d_{h, v_{j}} !}{d_{h, v_{j}}}\right.
$$

The contraction $\pi: C \rightarrow C_{0}$ of $e$ induces a contraction $\pi_{1}: C_{1} \rightarrow C_{2}$ and a tropical morphism $h^{\prime}: C_{2} \rightarrow C_{0}$ such that $\pi \circ h=h^{\prime} \circ \pi_{1}$. Moreover, all points in $h^{\prime-1}(\pi(q))$ lie in the interior of edges of $C_{2}$, except one point which is a trivalent vertex of $C_{2}$ (see figure 6 ). We also have

$$
|A u t(h)|=\left|A u t\left(h^{\prime}\right)\right|\left(d_{h, v_{1}}-2\right) ! \prod_{j=2}^{l} d_{h, v_{j}} !
$$

So if $C$ is rational, $\mathcal{L}=\emptyset$, and all points $p \in \mathcal{P}$ satisfy $v(q)=(2,1, \ldots, 1)$ except maybe two of them, by contracting all the points $q \in \mathcal{Q}$ with $v(q)=(2,1, \ldots, 1)$ we find ourselves in the situation discussed in [CJM]. In particular, we recover the same multiplicities in all cases except for example 1.5 (in this special case, the tropical computation from [CJM] is wrong). 
EXAMPLE 2.12. The result of this sequence of contractions in the case of the tropical morphism depicted in figure 2 a) is depicted in figure 6 .

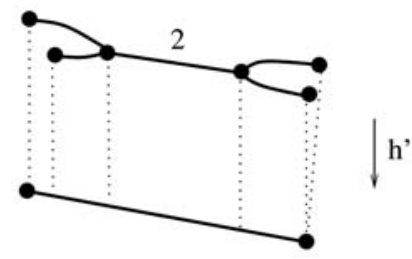

Fig. 6

\section{REFERENCES}

[BBM] B. Bertrand - E. Brugallé - G. Mikhalkin, Genus 0 characteristic numbers of the tropical projective plane. arXiv:1105.2004.

[CJM] R. CAVAlieri - P. Johnson - H. Markwig, Tropical Hurwitz numbers. J. Algebraic Combin. 32, no. 2 (2010), pp. 241-265.

[LZ04] S. LANDO - A. Zvonkin, Graphs on surfaces and their applications, volume 141 of Encyclopaedia of Mathematical Sciences. Springer-Verlag, Berlin, 2004. With an appendix by Don B. Zagier, Low-Dimensional Topology, II.

[Nis] T. Nishinou, Disc counting on toric varieties via tropical curves. arXiv:math.AG/0610660.

Manoscritto pervenuto in redazione il 28 settembre 2010. 
